\title{
Téoros
}

Revue de recherche en tourisme

\section{Les ambitions touristiques malmenées des municipalités} françaises

\section{La mise en valeur des églises et des chapelles rurales du Cantal et du Puy-de-Dôme}

\section{Fabien Venon}

Volume 23, numéro 2, été 2004

URI : https://id.erudit.org/iderudit/1071309ar

DOI : https://doi.org/10.7202/1071309ar

Aller au sommaire du numéro

Éditeur(s)

Université du Québec à Montréal

ISSN

0712-8657 (imprimé)

1923-2705 (numérique)

Découvrir la revue

Citer cet article

Venon, F. (2004). Les ambitions touristiques malmenées des municipalités françaises : la mise en valeur des églises et des chapelles rurales du Cantal et du Puy-de-Dôme. Téoros, 23(2), 48-53. https://doi.org/10.7202/1071309ar d'utilisation que vous pouvez consulter en ligne. 


\title{
Les ambitions touristiques malmenées des municipalités françaises
}

\author{
La mise en valeur des églises et des chapelles rurales \\ du Cantal et du Puy-de-Dôme
}

\section{Fabien Venon}

En France, la politique de sécularisation des biens du clergé, entamée à la Révolution, s'est achevée avec la Loi de séparation de l'Église et de l'État du 9 décembre 1905. Depuis, la charge du patrimoine religieux incombe à l'État et aux communes, circonscriptions administratives de base du pays. Les églises, laissées «à la disposition des fidèles et des ministres du culte pour la pratique de leur religion » (art. $5 \S 1$ de la Loi du 2 janvier 1907), deviennent un poids financier très lourd qui grève régulièrement le budget communal, notamment dans les campagnes dépeuplées auxquelles appartiennent les départements du Cantal et du Puy-de-Dôme (respectivement 26 et 76 habitants par kilomètre carré ; moyenne nationale: 108 habitants par kilomètre carré ; figure 1). La restauration d'une église dans le sud cantalien représente, par exemple, jusqu'à $10 \%$ du budget d'investissement annuel, de $2 \%$ à $4 \%$ sur une période de cinq ans. Les municipalités, élues par la population, envisagent donc de rentabiliser leurs investissements en développant l'activité touristique autour de leurs lieux de culte.

Le prestige d'une telle politique patrimoniale n'est pas négligeable car, «s'agissant du territoire, toute invocation d'un passé commun à son sujet-autrement dit d'un 'passé patrimonial' - est ipso facto articulée à la question de la légitimité du territoire en question (et prioritairement de son découpage) ainsi que de celle des autorités » (Micoud, 1999 : 55).

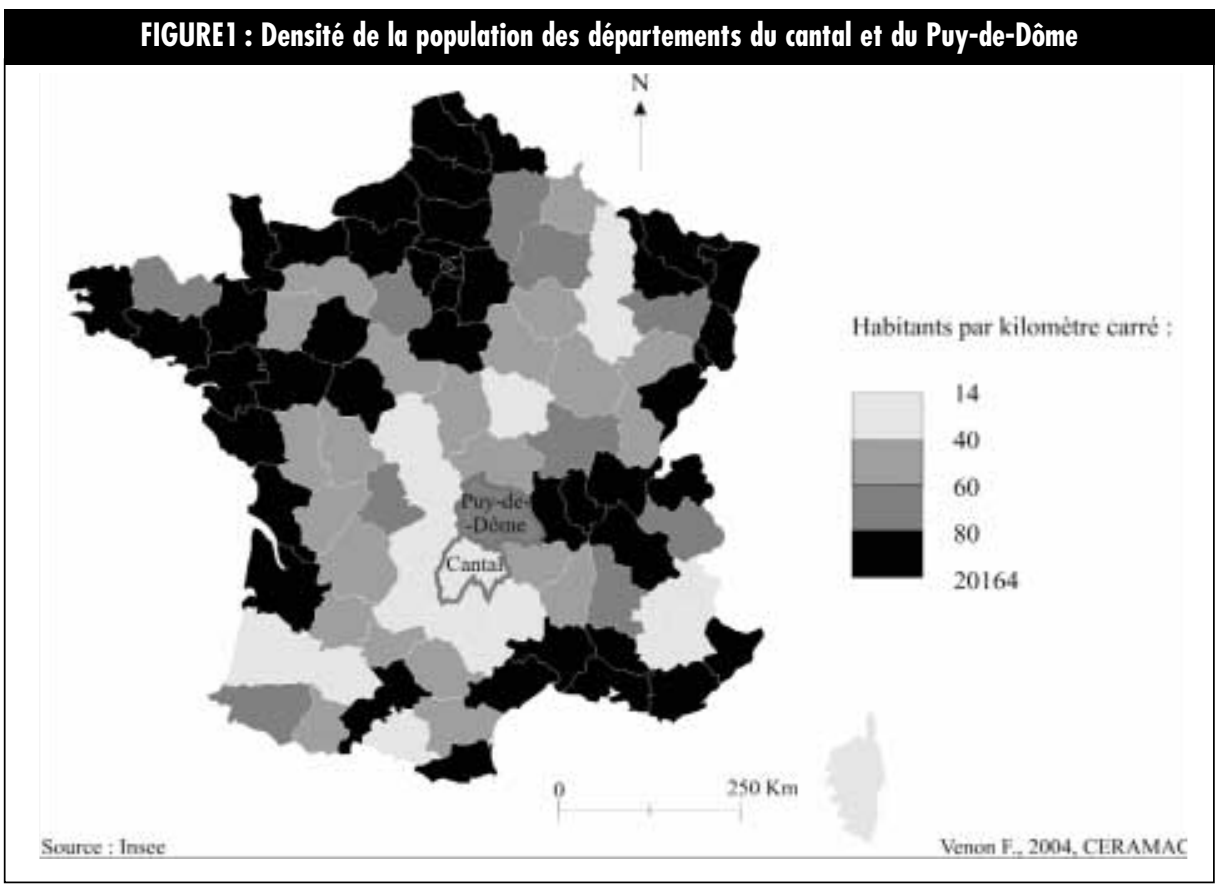

En somme, la mise en valeur du patrimoine religieux met en scène et donne corps à une communauté villageoise, souvent fragilisée par l'exode rural. Outre cet aspect identitaire, il s'agit surtout pour les communes de profiter d'un gisement potentiel de visiteurs non encore exploité économiquement. En 1987, $49 \%$ des Français ont visité, au moins une fois dans l'année, une église ou une cathédrale. Cette attitude ne cesse d'ailleurs de progresser. À cette date, $75 \%$ de nos compatriotes se déclarent amateurs d'art sacré contre $68 \%$ en 1980 (Mottura, 1993). De façon très significative, les Journées européennes du patrimoine, édition 2003, sont consacrées au patrimoine spirituel.

Ce genre d'initiatives est vivement encouragé. Consciente qu'un nombre considérable d'édifices religieux en Europe ne remplissent plus leurs fonctions originelles, l'assemblée parlementaire du Conseil de l'Europe, par sa résolution 916 ${ }^{1}$ adoptée le 9 mai 1989, «encourage une utilisation plus imaginative des édifices religieux existants » de la part des autorités responsables (églises, gouvernements et collectivités locales), tout en restant «compatible autant que possible avec l'intention qui a présidé à sa construction ». Une impulsion forte est donnée aux réaménagements civils, mais cette logique est exprimée en termes si diplomatiques que chacun peut y lire, au final, ce qu'il souhaite. Doit-on comprendre, par la volonté de sauvegarder les idéaux et les principes qui sont le patrimoine commun des États membres, que les nouvelles fonctions accordées aux lieux du culte doivent être nécessairement liées à la 
religion qui y trouvait demeure ? Ou, à l'exemple de la Scuola di San Pasquale à Venise, est-ce la dimension architecturale qui prime? Si tout paraît possible avec du temps et le soutien des communautés (article 8), peut-on toutefois qualifier les actions en faveur du tourisme religieux ou spirituel, menées sur l'initiative des municipalités, de tourisme durable?

\section{L'intercommunalité comme outil de développement du tourisme spirituel}

\section{La banalisation du patrimoine religieux rural}

Le village d'Albepierre-Bredons (237 habitants), situé dans le nord-est du Cantal, veille sur son territoire à la sauvegarde de deux édifices religieux : l'église paroissiale d'Albepierre et l'église monacale de Bredons. Le 22 août 1991, la foudre tombait sur le toit de l'église de Bredons, provoquant de nombreux dégâts. Dès 1989, les Monuments Historiques (M.H.) avaient envisagé des travaux sur les couvertures de cette église pour un montant d'environ 300 000 euros. Bien qu'elle était classée et qu'elle bénéficiait, de ce fait, de $75 \%$ de subventions, que les assurances versaient environ 35000 euros, la dépense restait élevée pour la commune, propriétaire de l'édifice. Au même moment, l'église du bourg d'Albepierre devait, elle aussi, être restaurée. Un programme de travaux intérieurs et extérieurs pour un montant d'environ 76225 euros avait été adopté par la municipalité. Le 11 août 1990, le couronnement du retable baroque de l'église d'Albepierre s'effondrait. L'estimation de la dépense pour la consolidation et la restauration du retable, qui n'était ni classé ni inscrit à l'inventaire supplémentaire, s'élevait à 34350 euros supplémentaires. La pression exercée par les M.H. pour faire les travaux à Bredons devenait de plus en plus forte, si bien que la charge globale d'entretien des édifices cultuels s'annonçait lourde pour la commune. Celle-ci a eu alors deux objectifs.

Le premier était d'obtenir le classement aux M.H. du retable d'Albepierre pour qu'il puisse bénéficier du maximum de subventions, en contrepartie d'un en- gagement financier aux travaux de Bredons. L'inscription à l'inventaire s'est faite le 10 décembre 1990 et le classement le 9 novembre 1993. Le second objectif était d'obtenir du curé desservant, résidant à Murat, l'autorisation de percevoir un droit d'entrée pour la visite de l'église de Bredons, en périodes de vacances scolaires, afin de trouver un financement pour l'entretien futur de cette église et de son mobilier. Après l'orage dévastateur du 22 août 1991, la commune a rapidement reçu l'accord du prêtre. Par la force des choses, l'activité touristique a donc pris pied dans le bâti religieux. Toutefois, très rapidement, la municipalité a compris qu'une commune isolée ne pouvait aujourd'hui mener à bien une politique touristique viable.

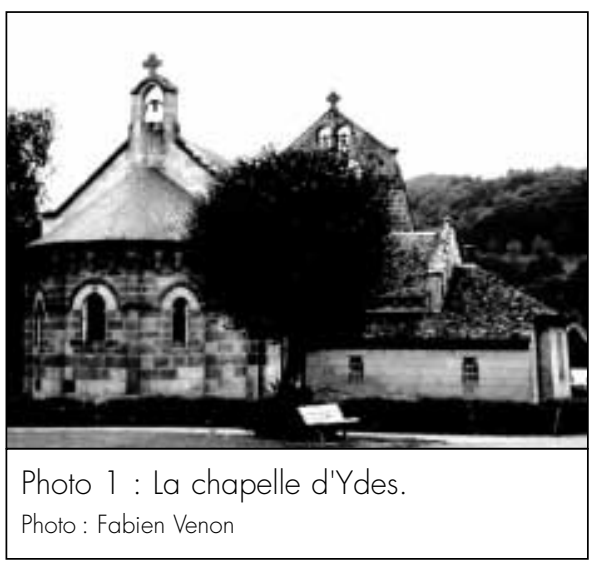

Le maillage communal français est le plus émietté d'Europe. La moitié des circonscriptions de l'Union européenne se situent dans ce pays. Dans ce cadre restreint, les moyens financiers et les forces vives deviennent de plus en plus difficiles à rassembler. Le plus difficile obstacle à briser est, notamment, l'enfermement du patrimoine religieux dans la banalité du quotidien. Seules des structures intercommunales peuvent aujourd'hui porter des projets touristiques viables autour des églises et des chapelles. En effet, c'est en transgressant leurs frontières que les communes parviennent, paradoxalement, à renforcer la spécificité des lieux de culte. Ainsi, la communauté de communes du pays de Murat, au nord-est du Cantal, est aujourd'hui chargée de développer l'animation touristique sur son territoire et, notamment, autour de l'église de Bredons.
On a recruté un animateur et effectué une enquête dans la commune d'Albepierre-Bredons auprès de 73 vacanciers. Le patrimoine religieux, vu comme un atout touristique, apparaît par le biais de la messe dominicale pour $0,4 \%$ des enquêtés, à la toute fin d'une longue liste qui évoque tous les aspects naturels et humains du village. Ce n'est donc pas particulièrement sur le culte régulier que peut être axée la politique touristique. Étrangement, ce ne sera que difficilement sur l'architecture religieuse elle-même. En effet, il ressort de l'étude, au grand étonnement de son responsable, que seuls $3 \%$ des touristes sont attirés par le patrimoine local et les festivités qui s'y déroulent. Il en est de même pour tous les éléments humains du milieu local, qu'ils soient gastronomiques (2,4\% des citations) ou sportifs (1,9\%). En somme, l'accueil et le contact avec la population locale se révèlent rarement recherchés $(2,4 \%$ des visiteurs seulement) car la notion d'authenticité, c'est-à-dire d'exclusivité de l'émotion touristique, n'est perçue que par $3 \%$ des touristes. Avec l'homogénéisation du genre de vie rural sur les standards urbains, tant culturels (alimentation, vêtements, loisirs...) que paysagers (trottoirs, éclairage, mobilier public...), c'est l'ensemble du patrimoine rural qui se banalise. Les diverses campagnes promotionnelles sont en partie responsables de cet état de fait puisqu'elles utilisent en toutes occasions une église stylisée comme symbole communal. De ce fait, les vacanciers sont exclusivement attirés par le cadre naturel ( $21 \%$ pour la campagne, les grands espaces et l'air pur, $18 \%$ pour le paysage). Il s'agit, par des promenades et des randonnées à proximité de la commune (13\%), de trouver le calme et la tranquillité $(26,5 \%)$, et le repos $(5 \%)$.

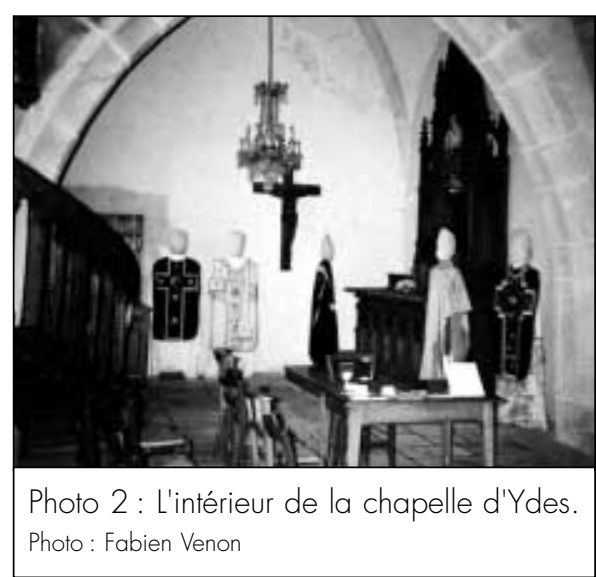


Toutefois, l'étude révèle aussi une très grande contradiction entre les attentes et les pratiques. En effet, interrogés sur les trois loisirs pratiqués durant leur séjour, les touristes déclarent les visites, donc un intérêt pour le patrimoine bâti, notamment religieux, à la hauteur de $57,2 \%$, en troisième position derrière le repos $(72 \%)$ et la marche $(97,2 \%)$. Conformément à l'intérêt naturiste du touriste, la marche domine toutefois largement le premier loisir déclaré avec $60 \%$ des citations, loin devant le repos $(24 \%)$ et les visites $(8 \%)$. C'est en deuxième et en troisième choix que les visites prennent toute leur importance avec respectivement $24,2 \%$ et $25 \%$ des citations. Le patrimoine bâti représente l'élément secondaire des excursions. Il ressort en définitive de cette étude que, dans leurs attentes, les vacanciers ne recherchent pas une animation particulière menée autour du patrimoine local, mais qu'ils visitent beaucoup de monuments au cours des promenades qu'ils effectuent. Il leur semble naturel de trouver, sur leur chemin de randonnée, les symboles du milieu rural tels qu'ils se le représentent, où l'église figure en premier plan, ce qui ne constitue en rien un motif d'attrait pour un territoire touristique particulier.

De ces constats découle la politique touristique du Pays de Murat. Il s'agit d'allier le goût naturel pour la randonnée et l'animation souhaitée d'un patrimoine délaissé autour d'un produit touristique reconnu comme exceptionnel. L'église de Bredons va donc adhérer à la Fédération des Sites Clunisiens, se distinguant de la masse par ce label apposé à l'entrée de la commune et sur le site même. Cette démarche achevée, un chemin de randonnée prendra place de Bredons à Marinval en Ardèche, ponctué par diverses traces artistiques laissées par les pèlerins. Ce produit est censé répondre aux attentes des randonneurs, premiers acteurs du tourisme local, tout en surfant sur la vague des chemins de pèlerinage de type Saint-Jacques-de-Compostelle. Un nouveau concept émerge : la randonnée à vocation spirituelle, dans laquelle le patrimoine religieux sert de toile de fond au développement d'un tourisme de nature.
Il ne faudrait pourtant pas croire que cette animation soit le fait d'une volonté particulière des autorités civiles, motivées par les propensions «budgétivores » des restaurations nécessaires au bâti. Le projet d'adhésion aux Sites Clunisiens provient d'une démarche de la fédération et non pas d'une demande de la municipalité d'Albepierre-Bredons. De même les activités culturelles, menées au sein de la chapelle, restent le fait des «Amis de Bredons ». Cette mainmise des associations sur le patrimoine est destinée à pallier le manque d'engagement des municipalités dans le domaine sensible qui touche au religieux.

\section{Les structures intercommunales : un simple relais des associations patrimoniales}

Le SIVOM ${ }^{2}$ de Chalus-Gignat-Villeneuve, dont la vocation tient à la restauration de la chapelle Sainte-Madeleine, sise sur la commune de Chalus, ainsi qu'à l'aménagement de ses abords, illustre l'un des rares exemples d'organisation intercommunale spécialisée dans l'animation culturelle d'un site religieux. Or, cette structure est née, non pas comme un centre d'actions à mener, laissées au libre arbitre de l'association des « Amis de Sainte-Madeleine », mais seulement comme un outil pour récolter des subventions.

Datée du dixième siècle, la chapelle de Sainte-Madeleine, située dans le hameau aujourd'hui disparu de Auzat sous Chalus, a servi d'église paroissiale aux villages de Chalus (doté seulement d'une chapelle castrale), de Gignat (pourvu d'une chapelle monacale) et de Villeneuve-Lembron (dont l'église actuelle date du quatorzième siècle) et constitue de ce fait un patrimoine commun aux trois communes (photo 3 ), malgré la présence d'une relique, le bras de Sainte-Madeleine, dès avant la Révolution ; les villageois des environs l'ont délaissée à mesure que leur étaient ouvertes les portes des chapelles monacale et castrale. La vente des biens du clergé, le 31 juillet 1791, et la raréfaction des cérémonies ont entériné la situation jusqu'à la désaffection complète de 1903. Complètement abandonnée depuis le début du siècle, la chapelle a subi de nombreux et réguliers pillages : colonnettes et chapiteaux arrachés, dalles enlevées, destruction du clocher et des couvertures. On a envisagé une première tentative de sauvegarde dès 1956 , avec une proposition d'inscription à l'Inventaire des Monuments Historiques (non obtenue). Près de trente ans se sont écoulés avant la naissance de l'association des «Amis de Sainte-Madeleine» dont les objectifs visaient: la sauvegarde, la restauration, la protection et les toutes activités culturelles sur le site. La présidente, infatigable «quêteuse » de cotisations et de main-d'œuvre, ralliant à sa cause les maires des trois communes concernées, mais aussi le sous-préfet d'Issoire et de nombreux gradés de l'École militaire d'Issoire dont les élèves seront détachés au débroussaillement du terrain, très vite, s'est rendu compte des difficultés que rencontre une association dans sa quête de subventions. C'est ainsi qu'est né le SIVOM C-G-V en septembre 1985, non pas de l'action motivée des municipalités, mais sous l'impulsion de la très influente présidente de l'association.

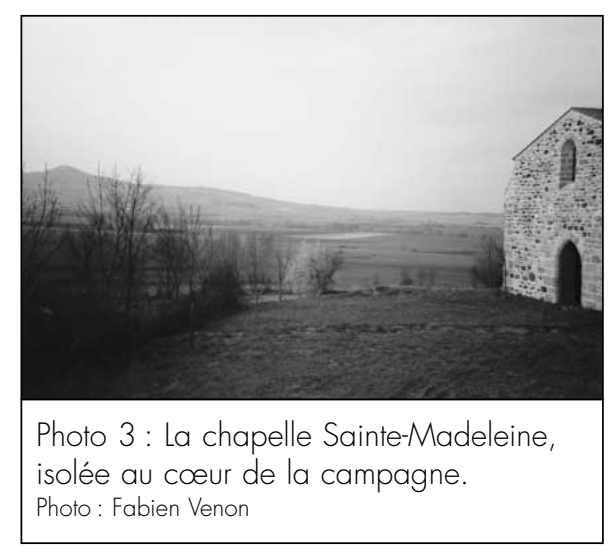

De fait, la naissance de cette structure intercommunale résulte, en premier lieu, du profond désintérêt, pour une chapelle en ruines, de la municipalité de Chalus, village médiéval doté par ailleurs d'un très riche patrimoine. La propriété du bâti et du terrain a été transférée au SIVOM et, par la suite, les trois municipalités se sont engagées très peu financièrement, puisque leur contribution au budget du syndicat, obligatoire au terme de l'article VII du statut, restait marginale (moins de 300 euros au total). La création d'un SIVOM relève du seul intérêt financier de l'association. 
La campagne des investissements 2001 correspondait à 18233 euros de travaux, auxquels il a fallu trouver un financement diversifié, la chapelle ne bénéficiant pas des subventions accordées par la DRAC (Direction régionale des Affaires culturelles), puisqu'elle n'est ni inscrite ni a fortiori classée. Un total de 5415 euros, soit $30 \%$ des fonds, émanait du Contrat de développement rural mis en place par le département du Puy-de-Dôme ; 4537 euros, soit $25 \%$ des recettes provenaient de la Dotation globale d'équipement ; 1417 euros (8\%) représentaient le remboursement de la taxe sur la valeur ajoutée (TVA) des campagnes menées en 1999; 1058 euros (6\%) correspondaient au remboursement des assurances à la suite des dégâts causés par la tempête de 1999; enfin, les 5806 euros (32\%) restants venaient des activités de l'association. On voit rapidement l'intérêt financier d'une telle structure. Les subventions ont atteint un niveau record avec $55 \%$ des fonds d'origine nationale ou départementale, sans compter le bénéfice tiré du remboursement quasi intégral de la TVA dont bénéficient les SIVOM.

Si le SIVOM a montré tout son intérêt quant à sa première mission de restauration du bâti, le manque d'engagement du syndicat dans le deuxième objectif, l'animation culturelle et les loisirs, se révèle aujourd'hui fortement préjudiciable au développement du site. En effet, les festivités, jusqu'alors organisées par l'association des Amis de Sainte-Madeleine, servent uniquement à récolter des fonds pour alimenter les budgets de restauration. L'amateurisme est de règle et l'accueil, rudimentaire. De plus, quelle pérennité aura l'association des Amis de Sainte-Madeleine une fois sa présidente disparue? Le SIVOM devra alors prendre ses responsabilités et une nouvelle ère s'ouvrira. Après n'avoir été qu'un outil dans la restauration du site, il devra, sous peine d'un retour de la chapelle à l'abandon, planifier une véritable politique d'animation culturelle. Or, cette seconde étape nécessite de proposer au public un produit d'exception pour lequel il serait prêt à payer, alors que toutes les autres églises de France sont ouvertes à titre gratuit. N'est-ce pas là le plus difficile ?

\section{Trouver un public pour le tourisme spirituel}

\section{Le patrimoine religieux est toujours associé à un usage gratuit}

Les maires des douze communes du canton de Saignes ont accepté de confier les objets cultuels de l'ancienne liturgie, souvent d'une grande beauté, à la salle du Trésor d'Ydes-Bourg sous forme de prêt. Si le trésor est installé dans l'ancienne sacristie de l'église d'Ydes-Bourg et non au chef-lieu cantonal, c'est en raison de l'aura du sénateur-maire d'Ydes, ancien président du Conseil général du Cantal, mais aussi du fait de la configuration favorable de la commune. Ydes, deuxième centre industriel du Cantal, cité laborieuse de 2026 habitants, est constituée de deux pôles bien distincts, mais complémentaires : Ydes-Centre, héritière d'un passé minier, autour de son église du dix-neuvième siècle et Ydes-Bourg, berceau de la commune, modeste village ramassé autour de son église romane du douzième siècle. $\mathrm{La}$ sacristie du dix-neuvième siècle, sans valeur architecturale, trouve un développement touristique: des vêtements sacerdotaux, une vierge, une pyxide, des calices, des croix, des reliquaires sont aujourd'hui exposés et protégés dans des vitrines munies d'un système anti-effraction et sous alarme permanente (photos 1 et 2). Cette réalisation a été encouragée par les prêtres desservants et par l'évêque :

\section{Je félicite le Sénateur Maire d'Ydes et je le remercie d'avoir constitué dans la sacristie de l'église d'Ydes Bourg, un Trésor. Ainsi notre patrimoine re- ligieux est non seulement conservé mais il est mis en valeur et il té- moigne d'un passé où nos compatri- otes estimaient juste et noble d'inves- tir dans ces objets sacrés et dans la beauté pour manifester leur foi et le sens du divin dont nos générations- même si elles les expriment autrement - ont également grand besoin ${ }^{3}$.}

Favorisée par ce consensus, la fréquentation crô̂t régulièrement depuis la première année où 300 personnes étaient accueillies. Durant l'été 2002, on a dé-

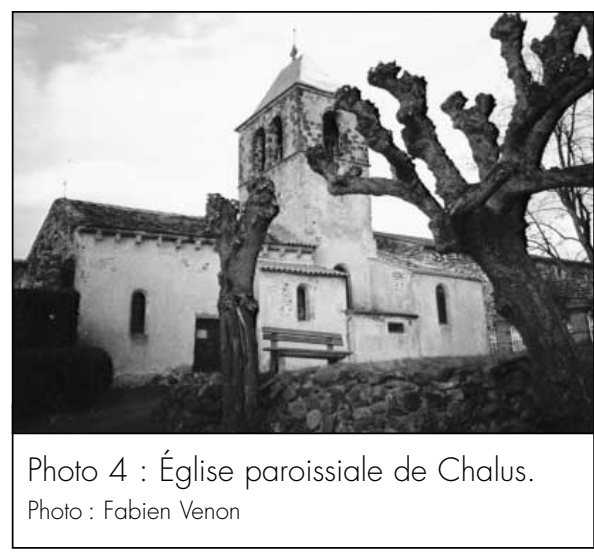

compté 571 visiteurs payants au tarif de deux euros, soit un chiffre d'affaires d'un peu plus de 1000 euros. Compte tenu des frais de gardiennage et d'accueil (un saisonnier), la fréquentation reste cependant trop faible pour équilibrer les charges. Durant cette saison, le nombre de visiteurs de l'église est estimé à 4000. Seul un visiteur sur sept est donc aujourd'hui prêt à payer pour approfondir ses connaissances en art religieux.

Pourtant, ce marché potentiel de l'amateur d'art religieux encourage le SIDET $^{4}$ à proposer, chaque mardi au départ d'Ydes, un circuit nocturne en car, accompagné d'un guide conférencier, de treize églises inscrites ou classées. Mis en place en 1999, il a rassemblé cette année-là 33 personnes en moyenne durant huit semaines. L'année suivante, la fréquentation s'est effondrée avec seulement 18 participants. En 2001, l'étiage était atteint avec 10 visiteurs. Aussi en 2002, au circuit purement religieux a-t-on associé le château de Trancis où une halte rafraîchissante est effectuée. Sur une saison raccourcie à six semaines, le nombre de participants a doublé, s'élevant à 20. L'évolution négative des effectifs laisse planer le doute sur la pérennité de ce produit touristique. Le public intéressé par l'art religieux, prêt à payer pour en profiter dans le cadre de visites organisées, est limité. Le patrimoine religieux est étroitement associé, dans l'esprit du grand public, à l'idée de gratuité et de libre accès. De fait, à long terme, des projets d'envergure bâtis autour du fait religieux s'effondrent souvent, faute d'un public suffisant. 


\section{L'animation touristique du patrimoine religieux est peu associée à la spiritualité}

Tel est le sort du musée des Pénitents Blancs de Marsac-en-Livradois, installé dans une chapelle datant du douzième siècle et inscrite sur la liste supplémentaire des Monuments Historiques (photo 5). À l'origine de la création surgissent trois notables locaux, dont le conservateur et restaurateur du Moulin à papier Richard de Bas. Sous le regard bienveillant de l'abbé Tourlonias, et à l'image de ce qui s'était construit autour des moulins à papier, ils souhaitent conserver une trace d'une tradition fortement ancrée à la terre du Livradois. Les premières associations, dites de Pénitents, sont nées au treizième siècle, prenant sur elles les péchés des fidèles, alors que l'Église catholique cessait d'imposer l'usage des pénitences publiques. Au début du dix-septième siècle, lorsque des confréries de Pénitents se sont implantées dans les monts du Livradois, sous l'impulsion de la Compagnie des Pénitents Blancs de Lyon, les tâches qu'elles s'assignaient témoignaient d'une notable évolution en répondant essentiellement à deux préoccupations, la piété et la charité, assurant une large reconnaissance auprès de la population. Lorsque, à la fin du dix-neuvième siècle, ces nobles objectifs ont disparu et que les assemblées et les processions ne sont devenues que de simples réunions d'amis, les confréries ont rapidement disparu, de même que la spectaculaire procession du Jeudi saint, au cours de laquelle la population assistait à une évocatrice flagellation du Christ portant sa croix. Ne restait de traces qu'une chapelle, en partie détruite, ruinée par l'humidité, qui servait, depuis un demi-siècle, de débarras ou de remise.

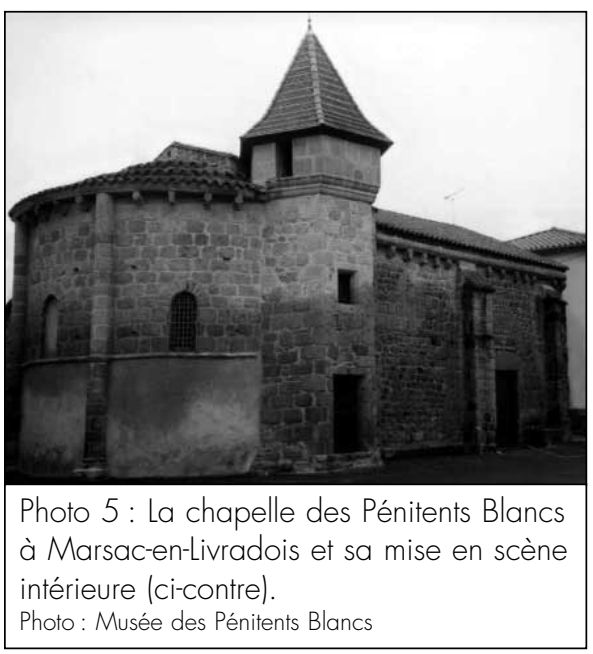

Bien que les pénitents soient ancrés dans l'imaginaire local, mais aussi national et européen, la fréquentation de ce musée décline irrémédiablement. De l'inauguration du musée le 14 juin 1958 jusqu'en 1989 , plus de 120000 visiteurs sont recensés, soit une moyenne de 4000 personnes par an. Aujourd'hui, les derniers décomptes font état de moins de 800 visiteurs (tableau 1). Comment expliquer une telle désaffection? La fréquentation a été divisée par deux depuis la fermeture du restaurant local en 2000. En effet, la présence d'un point de restauration permettait l'intégration du musée aux circuits touristiques organisés par les caristes qui, depuis, se sont détournés de la commune. L'attrait propre de ce musée se révèle donc fort limité, puisqu'il ne survit que par l'apport des autres points forts touristiques de la région qui, réunis, peuvent justifier une journée complète de visites. Isolé, il n'imprime pas de dynamique propre, faute d'avoir une clientèle particulière. Pour compenser la diminution du public touristique, la chapelle des Pénitents ne peut, en effet, compter sur l'engagement du clergé, désormais absent des démarches communales ou intercommunales, ce qui se révèle préjudiciable à long terme. Les musées à caractère religieux et les récitals de musique sacrée dans les églises se privent ainsi de l'extraordinaire moyen de promotion qu'est la communauté des croyants. Étrangement, ce musée ne connaît la visite d'une classe d'école catholique qu'une fois tous les cinq ans.
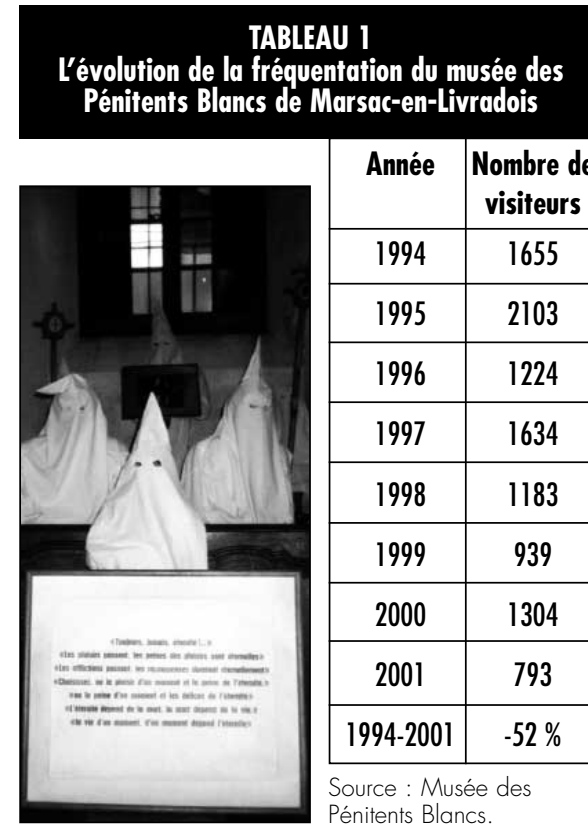

\begin{tabular}{|c|c|}
\hline Année & $\begin{array}{c}\text { Nombre de } \\
\text { visiteurs }\end{array}$ \\
\hline 1994 & 1655 \\
\hline 1995 & 2103 \\
\hline 1996 & 1224 \\
\hline 1997 & 1634 \\
\hline 1998 & 1183 \\
\hline 1999 & 939 \\
\hline 2000 & 1304 \\
\hline 2001 & 793 \\
\hline 1994-2001 & $-52 \%$ \\
\hline
\end{tabular}

Pourtant, quelques grandes tendances laissent entrevoir l'intérêt d'un développement de produits touristiques à forte identité religieuse. D'une part, les autorités publiques doivent régulièrement amadouer les susceptibilités de la population catholique. Comme l'affirme Michel Moncault, vicaire général du diocèse de Fréjus-Toulon et expert à la Commission épiscopale de liturgie, «il ne faut jamais oublier que, même vide, l'église reste maison de Dieu et de la communauté» (Penez, 2000: 36). Un partenariat étroit avec le clergé est très souvent à l'origine des projets (Bredons, Ydes, Marsac-en-Livradois) et l'effondrement du nombre de visiteurs accompagne souvent la mise à l'écart du pouvoir religieux. D'autre part, la régression de la culture religieuse des jeunes générations (moins d'enfants baptisés et catéchisés) nécessite un énorme effort pédagogique. Aujourd'hui, la demande d'enseignement du fait religieux en dehors des institutions religieuses et, notamment, dans l'école publique, s'accroît face à

la menace de plus en plus sensible d'une déshérence collective, d'une rupture des chaînons de la mémoire nationale et européenne où le maillon manquant de l'information religieuse rend strictement incompréhensibles, voire sans intérêt, les tympans de la cathédrale de Chartres, La crucifixion $d u$ Tintoret, le Don Juan de Mozart, le Booz endormi de Victor Hugo, et la Semaine sainte d'Aragon (Debray, 2002 : 13-14).

Toutefois, la dimension religieuse du lieu et des collections présentées ne doit pas interférer avec un discours qui porterait atteinte à la laïcité. Fondée sur le célèbre «Rendez à César ce qui est à César et à Dieu ce qui est à Dieu », la laïcité à la française reconnaît officiellement la plus stricte séparation entre les actions de l'État et celles des institutions religieuses reconnues.

$\mathrm{Au}$ final, les collectivités locales ne paraissent pas les mieux placées pour développer ces projets à vocation spirituelle. L'amalgame civil et religieux pèse lourdement sur le déficit de fréquentation actuel. La puissance publique n'ose pas développer une communication d'envergure vers le public des croyants. La fréquentation stagne et s'épuise. Peut-on alors parler d'un développement touristique durable sur la base du patrimoine religieux ? $\mathrm{La}$ durabilité touristique nécessite, selon les 
principes proposés par l'Association Française d'Ingénierie Touristique, une amélioration de la vie de la communauté qui reçoit le touriste, une expérience de première qualité pour le visiteur, un maintien de la qualité de l'environnement sur lequel comptent à la fois le visiteur et la communauté d'accueil. Ces trois points conduisent, de prime abord, à un profond scepticisme.

D'une part, les exemples de développements volontaires portés par des municipalités se comptent sur les doigts d'une main. D'autre part, compte tenu du profond respect de la sensibilité religieuse de la communauté catholique et de la sacralité du lieu, les aménagements sont de faible ampleur et l'expérience vécue par le visiteur loin d'être ressentie comme unique. D'ailleurs, dans les lieux du culte encore en service, le développement économique de la commune ne saurait faire figure d'objectif. Les recommandations du Conseil permanent de l'Épiscopat français ${ }^{5}$ précisent : "c'est en raison même de leur caractère particulier de lieu de l'Alliance entre Dieu et les hommes que l'accès des églises doit rester libre et gratuit». Quelques rares dérogations peuvent être accordées par l'Ordinaire, mais, de toute manière, «le droit d'entrée ne pourra en aucun cas couvrir une opération purement commerciale». Par ailleurs, «les organisateurs n'oublieront pas de rembourser aux responsables de l'église les dépenses occasionnées par la tenue du concert : chauffage, électricité, entretien... », de même qu'ils devront «accorder aux artistes et aux musiciens la juste rémunération à laquelle ils ont droit ». L'organisation d'un concert a donc un coût sans pouvoir en tirer une forte rétribution (1940 euros de recettes contre 2215 euros de dépenses pour l'association des Amis du Prieuré, à Saint-Hilaire-la-Croix, en 2000). L'équilibre du budget est donc quasiment impossible dans des lieux du culte.

Aussi, les répercussions sur la région d'accueil semblent-elles pour l'heure bien faibles (ouverture estivale seulement, peu d'emplois directs ou indirects : un emploi saisonnier au mieux, pas d'intégration aux circuits touristiques locaux). La maitrise quantitative et qualitative de la fréquentation est d'ailleurs largement souhaitée par les aménageurs pour qui elle est une condition de la conservation du bâti. Pour la municipalité de Murat, «le village ne doit pas perdre son authenticité et son âme» (Lecoq, 1999: 14). Dans le même esprit, l'abbé Tourlonias, curé de Marsac-enLivradois, s'inquiète des possibles répercussions des flux touristiques sur la société locale. «En signalant un point d'histoire du Livradois, j'aurai témoigné ma tendresse pour ce divin coin de terre, envahi aujourd'hui par l'usine et, demain, encombré, peut-être sali par le tourisme tapageur» (Hemeret, 1989: 2). Tant pour les autorités religieuses que civiles, la vocation des aménagements s'inscrit principalement dans la restauration du site. Plus qu'à un développement touristique durable, nous faisons face à une conservation touristique durable.

Dans l'avenir, il va falloir brusquer les sensibilités pour que les touristes, en particulier, comprennent qu'il est impossible de proposer une émotion exceptionnelle tant qu'ils n'accepteront pas des projets d'envergure, au sein même des lieux du culte ${ }^{6}$. Au préalable, un partenariat étroit devrait s'établir entre les pouvoirs publics et l'institution religieuse, auquel n'appelle pas l'actuelle interprétation de la laïcité française. Comme le note Danièle Hervieu-Léger (1998: 19), spécialiste du catholicisme français,

Confrontée à l'atomisation individualiste des revendications (y compris sur le terrain religieux), à la prolifération des microcommunautés et à la logique de la mondialisation, la laïcité doit prendre en charge, sur de nouveaux terrains, le travail d'élaboration des valeurs capables de fonder les solidarités vécues d'une société d'individus.

Pour cela, il conviendrait d'établir un consensus autour de cérémonies et d'animations culturelles, sportives ou touristiques, fédérant à de nombreuses occasions la population locale, les croyants et les touristes. Quand verra-t-on en France, exemple frappant d'utilisation mixte des édifices religieux, un gymnase prendre place dans la nef aux côtés d'un chœur réservé au culte, comme à l'église communale de Sainte-Françoise de Lotbinière (1931), petite commune de 450 habitants au Québec (Comité multisectoriel du patrimoine religieux de Portneuf, 2000) ? La tradition française ne conduit-elle pas, au contraire, à séparer les usages ? Les animations culturelles souhaitées par les pouvoirs publics ne sont-elles pas vouées à rester cloisonnées dans des lieux désaffectés du culte de longue date, comme en témoignent les exemples des chapelles de Marsac-en-Livradois ou de Chalus (photo 4)?
Fabien Venon est doctorant en géographie à l'université Blaise-Pascal (Clermont-Ferrand, France); il est attaché temporaire d'enseignement et de recherche à l'Université Blaise-Pascal.

\section{Notes}

1 [http://assembly.coe.int/Mainf.asp?link=http $\% 3 \mathrm{~A} \% 2 \mathrm{~F} \% 2 \mathrm{Fassembly}$.coe.int $\% 2 \mathrm{FDocum}$ ents $\% 2$ FadoptedText $\% 2$ FTA89\%2FFRES9 16.HTM].

2 Syndicat intercommunal à vocation multiple.

3 Discours d'inauguration du Musée d'Ydes par l'évêque de Saint-Flour, Mgr Séjourné.

4 Syndicat intercommunal de développement économique et touristique des cantons de Saignes et de Champs-sur-Tarentaine.

5 [http://www.portstnicolas.org/cms.php? pageid=377].

6 Lors de deux enquêtes menées en 2002 dans les églises d'Issoire et de Murat, un tiers des visiteurs estivaux s'opposent à toute affectation, même ponctuelle, d'une église en musée ou en salle de spectacle. Un répondant sur dix seulement envisage une affectation permanente.

\section{Bibliographie}

Penez, Catherine (dir.) (2000), Regards sur le patrimoine religieux, De la sauvegarde à la présentation, Paris, Actes Sud.

Comité multisectoriel du patrimoine religieux de Portneuf (2000), Actes du colloque sur l'avenir du patrimoine religieux du Portneuf, Un clocher, Un village, Cap-Santé, Municipalité régionale de comté (MRC) de Portneuf.

Debray, Régis (2002), L'enseignement du fait religieux dans l'école laïque, Rapport au ministre de l'Éducation nationale, Paris, Odile Jacob.

Hemeret, Georges et Jeanne (1989), L'envoûtant secret des pénitents blancs d'Auvergne, Marsac-enLivradois, Municipalité de Marsac-en-Livradois.

Hervieu-Léger, Danièle (1998), «Repenser la laïcité et le dialogue interreligieux », France Forum, n 231, juillet-septembre, p. 14-19.

Lecoq, Éric (1999), Albepierre-Bredons, ses visiteurs, Questionnaire été 1999, fascicule ronéotypé.

Micoud, André (1999), « Patrimoine et légitimité des territoires. De la construction d'un autre espace et d'un autre temps commun», dans F. Gerbaux (dir.), Utopie pour le territoire : cohérence et complexité ?, La Tour d'Aigues, Édition de l'Aube, p. 53-63.

Mottura, Pierre (1993), «Au seuil du troisième millénaire, vers un tourisme spiritualiste?», Cahier Espaces, n 30 , mars, p.154-160. 